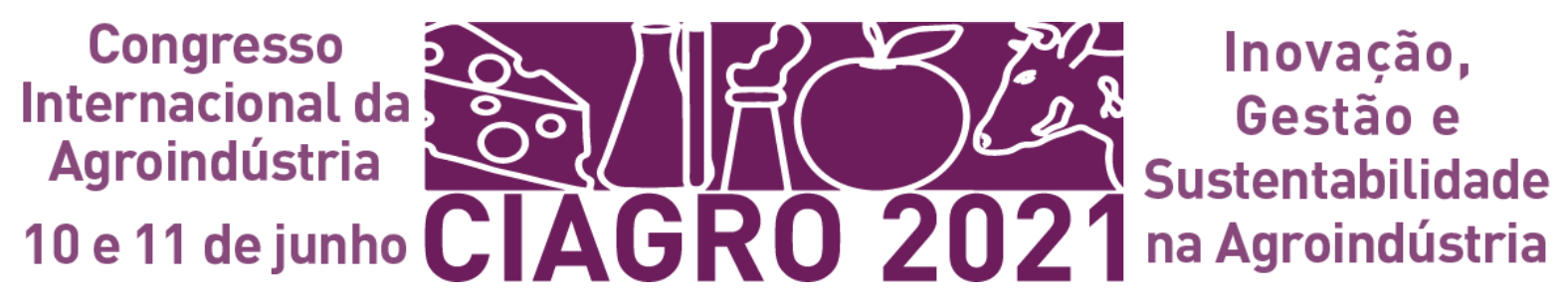

\title{
AGRICULTURA 4.0: UMA VISÃO GERAL DAS INOVAÇÕES TECNOLÓGICAS NO AUMENTO DA PRODUTIVIDADE AGRÍCOLA
}

\author{
AGRICULTURA 4.0: UNA VISIÓN GENERAL DE LAS INNOVACIONES \\ TECNOLÓGICAS PARA AUMENTAR LA PRODUCTIVIDAD AGRÍCOLA
}

\section{AGRICULTURE 4.0: AN OVERVIEW OF TECHNOLOGICAL INNOVATIONS IN INCREASING AGRICULTURAL PRODUCTIVITY}

\author{
Afonso Henrique da Silva Júnior ${ }^{1}$; Éllen Francine Rodrigues ${ }^{2}$; Lisandro Simão $^{3}$ Luís Antonio Lourenço $^{4}$; Carlos \\ Rafael Silva de Oliveira ${ }^{5}$
}

DOI: https://doi.org/10.31692/IICIAGRO.0137

\begin{abstract}
RESUMO
A agricultura 4.0 está atrelada às inovações tecnológicas de alta performance inseridas no segmento agrícola, em que proporcionam autonomia, flexibilidade, aumento de produtividade e entre outros pontos positivos ao produtor rural. Os pilares da agricultura 4.0 estão pautados na integração da computação, comunicação e controle por meio de redes e processos físicos, ou seja, os sistemas ciberfísicos. Nesse contexto, as tecnologias inseridas na agricultura 4.0 são a computação em nuvem, o aprendizado de máquina, a realidade virtual, a inteligência artificial e etc. E é através dessas ferramentas que é possível a obtenção de insights, modelos preditivos, reconhecimento de padrões, imagens de satélite, sensores, hardwares, softwares e entre outros para a visualização de maneira ampla as necessidades da propriedade e a realização de previsões da produtividade da cultura. Diante disso, o Brasil como um dos principais produtores de alimentos do mundo tem papel importante nesse progresso agrícola, pois sua adesão a essas tecnologias possibilitará aumentar a atual performance da produtividade agrícola e a modernização das lavouras em território nacional. Contudo, um grande desafio para a inserção dessas tecnologias inerentes à agricultura 4.0 é a disponibilidade de internet no campo, pois na maior parte é necessário a integração de computadores e sensores através desse recurso. Assim, a presente revisão objetiva abordar as diferentes ferramentas digitais presentes na agricultura 4.0 e como essas tecnologias podem auxiliar no aumento da produção agrícola ao longo dos anos, seja em aumento de produtividade e/ou na tomada de decisões diante dos desafios da lavoura. Por último, também foram apresentados os potenciais e as limitações da inserção dessas tecnologias no contexto do produtor rural brasileiro e de países emergentes.
\end{abstract}

Palavras-Chave: Tomada de decisão, Inteligência artificial, Big data, Aprendizado de máquina, Internet of things.

\footnotetext{
1 Engenheiro Agroindustrial-Agroquímico (Universidade Federal do Rio Grande - FURG), Mestrando em Engenharia Química (Universidade Federal de Santa Catarina - UFSC), afonso.silva@posgrad.ufsc.br

2 Engenheira de Alimentos (Universidade de Passo Fundo - UPF), Doutora em Engenharia Química (Universidade Federal de Santa Catarina - UFSC), ellenfrodrigues@ gmail.com

${ }^{3}$ Engenheiro Ambiental (Universidade do Extremo Sul Catarinense - UNESC), Doutor em Ciência e Engenharia de Materiais (Universidade Federal de Santa Catarina - UFSC), lisandrosimao@ gmail.com

4 Engenheiro Químico, Instituto Latino-Americano de Tecnologia, Infraestrutura e Território (ILATIT), Universidade Federal da Integração Latino-Americana (UNILA), luis.lourenco@unila.edu.br

${ }^{5}$ Engenheiro Têxtil (Universidade Estadual de Maringá - UEM), Doutor em Engenharia Química (Universidade Federal de Santa Catarina - UFSC), carlos.oliveira@ufsc.br
} 


\section{RESUMEN}

La agricultura 4.0 está ligada a innovaciones tecnológicas de alto desempeño insertadas en el segmento agrícola, donde proporcionan autonomía, flexibilidad, aumento de la productividad y, entre otros puntos positivos a los productores rurales. Los pilares de la agricultura 4.0 se basan en la integración de la informática, la comunicación y el control a través de redes y procesos físicos, es decir, sistemas ciberfísicos. En este contexto, las tecnologías insertadas en la agricultura 4.0 son la computación en la nube, el aprendizaje automático, la realidad virtual, la inteligencia artificial, etc. Y es a través de estas herramientas que es posible obtener insights, modelos predictivos, reconocimiento de patrones, imágenes satelitales, sensores, hardware, software y otros, para una visión amplia de las necesidades de las propiedades y la realización de pronósticos de la productividad de la cultura. Ante esto, Brasil como uno de los principales productores de alimentos del mundo tiene un papel importante en este avance agrícola, ya que su adhesión a estas tecnologías permitirá incrementar el desempeño actual de la productividad agrícola y la modernización de los cultivos en el territorio nacional. Sin embargo, un gran desafío para la inserción de estas tecnologías inherentes a la agricultura 4.0 es la disponibilidad de internet en el campo, ya que en la mayoría de los casos es necesario integrar computadoras y sensores a través de este recurso. Así, esta revisión tiene como objetivo abordar las diferentes herramientas digitales presentes en la agricultura 4.0 y cómo estas tecnologías pueden ayudar a aumentar la producción agrícola a lo largo de los años, ya sea en el aumento de la productividad y/o en la toma de decisiones frente a los desafíos de los cultivos. Finalmente, también se presentaron las potencialidades y limitaciones de la inserción de estas tecnologías en el contexto de productores rurales en Brasil y en países emergentes.

Palabras Clave: Toma de decisiones, Inteligencia artificial, Big data, Machine learning, Internet de las cosas.

\section{ABSTRACT}

Agriculture 4.0 is linked to high-performance technological innovations inserted in the agricultural segment, in which they provide autonomy, flexibility, increased productivity, and, among other positive points, to rural producers. The pillars of agriculture 4.0 are based on the integration of computing, communication, and control through networks and physical processes, that is, cyberphysical systems. In this context, the technologies inserted in agriculture 4.0 are cloud computing, machine learning, virtual reality, artificial intelligence, etc. And it is through these tools that it is possible to obtain insights, predictive models, pattern recognition, satellite images, sensors, hardware, software, and others, to broadly visualize the needs of the property and to make forecasts of the productivity of the crops. Given this, Brazil as one of the main food producers in the world has an important role in this agricultural progress, as its adherence to these technologies will make it possible to increase the current performance of agricultural productivity and the modernization of crops in the national territory. However, a major challenge for the insertion of these technologies inherent to agriculture 4.0 is the availability of the internet in the field, since in most part it is necessary to integrate computers and sensors through this resource. Thus, this review aims to address the different digital tools present in agriculture 4.0 and how these technologies can increase agricultural production over the years, whether in increasing productivity and/or in decision-making in the face of crop challenges. Finally, the potentials and limitations of the insertion of these technologies in the context of rural producers in Brazil and emerging countries were also presented.

Keywords: Decision making, Artificial intelligence, Big data, Machine learning, Internet of things.

\section{INTRODUÇÃO}

De acordo com a previsão da Organização das Nações Unidas (ONU), a população mundial atingirá a marca de no mínimo 9,5 bilhões de habitantes no ano de 2050 (ANGOM; VISWANATHAN; RAMESH, 2021). Essa expectativa pode ser superada, devido ao rápido crescimento populacional ao longo dos últimos anos em países emergentes, por exemplo, na Índia. Nos últimos 10 anos, a Índia passou de 1,234 bilhão de habitantes para 1,366 bilhão, 
um aumento de mais de 100 milhões de pessoas (SAHU et al., 2020). E isso também é perceptível em outras nações, o que vem causando preocupação às entidades mundiais, principalmente no que diz respeito à demanda de alimentos daqui 30 anos 2050 (ANGOM; VISWANATHAN; RAMESH, 2021). Segundo a Organização das Nações Unidas para a Alimentação e a Agricultura (FAO), a disponibilidade de terras agrícolas está localizada em poucos países e somente na América Latina, há a possibilidade de expansão de áreas para o cultivo de aproximadamente 90\% (BJORNLUND; BJORNLUND, 2019; FUENTESLLANILLO et al., 2021). O Brasil, por exemplo, é um dos produtores mais importantes do mundo e tem grande potencial de aumentar exponencialmente a sua oferta de alimentos (ZILLI et al., 2020).

A agricultura em território nacional vem apresentando alterações com o passar do tempo, principalmente com relação à expansão de terras destinadas para o plantio de commodities (FUENTES-LLANILLO et al., 2021). Contudo, por causa de restrições e a inserção de novas tecnologias no campo, organizações apostam na possibilidade de aumentar a produção agrícola com o espaço que se utiliza hoje em dia. O progresso científico relacionado ao mundo do agronegócio e principalmente associado aos esforços de aumento da produção podem ser visualizados em diferentes vertentes, como na evolução da genética e das técnicas agronômicas. Apesar disso, existem desafios envolvidos no que diz referência aos estudos genéticos, por exemplo, alto custo e demanda altamente qualificada de profissionais da área. Diante disso, as tecnologias habilitadoras dentro do contexto de agricultura 4.0 vêm se tornando uma aliada no desafio do aumento da produção agrícola (JUNG et al., 2021).

As tecnologias habilitadoras estão presentes em diferentes cenários, seja na indústria de transformação para a automatização do processo produtivo ou no cotidiano das pessoas que utilizam ferramentas digitais para a análise de dados. Além disso, essas tecnologias estão associadas principalmente à otimização do tempo, na redução de custos e na tomada de decisões (KAKANI et al., 2020). Os pilares da agricultura 4.0 estão pautados na integração da computação, comunicação e controle por meio de redes e processos físicos, ou seja, os sistemas ciberfísicos.

De acordo com o Instituto Nacional de Padrões e Tecnologia (NIST), esses sistemas são capazes de combinar o mundo físico com o cibernético, utilizando ferramentas digitais que podem responder em tempo real aos seus respectivos ambientes (AFOLALU et al., 2021). Dentre essas tecnologias estão a computação em nuvem, o aprendizado de máquina, a realidade virtual, a inteligência artificial e entre outras ferramentas (BALDUCCI; IMPEDOVO; PIRLO, 2018). Com isso, através de insights, modelos preditivos, 


\section{AGRICULTURA 4.0: UMA VISÃO GERAL DAS INOVAÇÕES TECNOLÓGICAS}

reconhecimento de padrões, imagens de satélite, sensores, hardwares, softwares e entre outros é possível visualizar de maneira ampla as necessidades da propriedade e a produtividade da lavoura (TALAVIYA et al., 2020). Portanto, compreende-se o quanto é positivo a aplicação desses conceitos na agricultura contemporânea.

Diante do exposto anteriormente, a presente revisão objetiva abordar as diferentes ferramentas digitais presentes na agricultura 4.0 e como essas tecnologias podem auxiliar no aumento da produção agrícola ao longo dos anos, seja em aumento de produtividade e/ou na tomada de decisões diante dos desafios da lavoura. Por último, também foram apresentados os potenciais e as limitações da inserção dessas tecnologias no contexto do produtor rural brasileiro e de países emergentes.

\section{AGRICULTURA 4.0 E AS TECNOLOGIAS HABILITADORAS}

O termo agricultura 4.0 está atrelado às inovações tecnológicas de alta performance inseridas no segmento agrícola, em que proporcionam autonomia, flexibilidade, aumento de produtividade e entre outros pontos positivos ao produtor rural. No entanto, para o melhor entendimento desse termo dentro da agricultura é fundamental conhecer um pouco sobre a evolução do cenário agrícola que está acompanhado ao progresso da humanidade (JONES et al., 2017).

Há 10 mil anos atrás, ainda quando a sociedade era nômade e vivia apenas da caça e dos frutos que encontrava pelas florestas, não via a necessidade de produzir seu próprio alimento. Contudo, com a escassez de comida, o desenvolvimento de ferramentas auxiliares para o ofício do dia a dia e o aprimoramento das primeiras impressões sobre o manejo do solo, começaram a formação de grupos sedentários. Esses grupos ao longo dos anos perceberam que era melhor se fixarem nas proximidades de rios, pois tinham próximo um elemento essencial para a vida e para o cultivo, a água. Além disso, possuía também por perto terras férteis, favorecendo a agricultura. Com o passar do tempo, o ser humano começou a acumular conhecimento sobre o processo de semeadura, o uso de ferramentas e as influências do meio externo sobre a produção agrícola (POLOZ, 2021). Mas, ainda permaneceram por muitos séculos "parado" no tempo, utilizando como força de tração animais (cavalos, bois e entre outros) e o cultivo era realizado manualmente, limitando a produtividade da lavoura (AFOLALU et al., 2021).

Isso tudo começou a mudar somente no século XVIII, quando ocorreu a Primeira Revolução Industrial. Nesse período foi possível visualizar grandes veículos a vapor nos campos, promovendo os primeiros eventos rumo à mecanização da agricultura (POLOZ, 
2021). Já na Segunda Revolução Industrial, no século XIX, com o avanço das pesquisas científicas relacionadas à fertilização e proteção das plantas juntamente com os motores a combustão, começaram de fato a evolução agrícola até o que conhecemos hoje, com a inserção de tecnologias digitais para a otimização da produção (AFOLALU et al., 2021). Assim, com a adoção de recursos computacionais e decisões baseadas na análise de dados, iniciou-se a agricultura 4.0, o que está sendo vivenciado hoje em dia.

\section{Big data}

Big data é geralmente referido a uma coleta muito grande de dados ou informações que não podem ser definidas ou de certa forma descrita precisamente em alguma linguagem computacional. O big data pode ser descrito como os cinco "V"s (KAMILARIS; KARTAKOULLIS; PRENAFETA-BOLDÚ, 2017):

- Volume: diz respeito ao tamanho dos dados coletados para que seja realizado a análise;

- Velocidade: os dados são gerados com muita rapidez, ou seja, a velocidade é o intervalo de tempo em que esses dados são úteis e relevantes. Uma aplicação na agricultura que está relacionado à velocidade está na identificação de pragas, pois devem ser analisados com muita rapidez para que seja tomada uma decisão no que tange esse problema;

- Variedade: os dados podem ser advindos de diversas fontes e em diferentes tempos, tais como imagens, vídeos, áudios, documentos em geral, planilhas e entre outros. Além disso, em diferentes domínios de aplicação, assim são necessárias a identificação e a melhor forma de serem armazenados;

- Veracidade: está relacionado à qualidade e confiabilidade dos dados, porém, com a análise estatística desse grande volume de informações pode-se compensar as informações "incorretas" que estão armazenadas; e

- Valor: a geração de valor a partir do volume de dados, ou seja, a capacidade de propagar o conhecimento/inovação.

Apesar do big data ser descrito por diferentes pesquisadores como os cinco "V"s mencionados acima, não necessariamente precisa satisfazer todas essas cinco dimensões (KAMILARIS; KARTAKOULLIS; PRENAFETA-BOLDÚ, 2017). A sua aplicação no segmento agrícola o torna fundamental nas tomadas de decisões, porém, há a necessidade de grandes investimentos em processamento de dados e de espaço para armazenamento. Isso, 


\section{AGRICULTURA 4.0: UMA VISÃO GERAL DAS INOVAÇÕES TECNOLÓGICAS}

porque deve ser aplicado na maioria das vezes em tempo real para diversas situações dentro da lavoura, por exemplo, a previsão do tempo (TRIPATHI; SRINIVAS; NANJUNDIAH, 2006). Nas bases científicas encontra-se diversos trabalhos em que aplicam técnicas de análise de big data na agricultura. Na Tabela 01 mostra alguns desses estudos e a descrição do problema analisado.

Tabela 01: Lista de alguns trabalhos encontrados na literatura em que utilizaram técnicas de análise de big data aplicados às problemáticas da agricultura.

Problemática Título do trabalho

Previsão das mudanças climáticas

Downscaling of precipitation for climate change scenarios: a support vector machine approach

Superior fuzzy enumeration crop prediction algorithm for big data agriculture applications produtividade das culturas

O uso do big data para a visualização e análise de dados na ciência climática

Caracterização de solos e plantas

Os efeitos nos oxidantes autotróficos de amônia em solos agrícolas
MERRA Analytic Services: meeting the big data challenges of climate science through cloud-enabled climate analytics-as-a-service

Intensified fuzzy clusters for classifying plant, soil, and residue regions of interest from color images

Investigations of soil autotrophic ammonia oxidizers in farmlands through genetics and big data analysis
Referência

(TRIPATHI

SRINIVAS; NANJUNDIAH, 2006)

(VELMURUGAN;

KANNAGI;

VARSHA, 2021)

(SCHNASE et al., 2017)

(MEYER et al., 2004)

(ZHANG; GUAN; JIANG, 2021)

Fonte: Autores, (2021).

Para a análise de big data são requeridas diversas técnicas e abordagens, tais como aprendizado de máquina, análise estatística, plataformas em nuvem, modelagem, análise geoespacial, processamento de imagens e entre outros. E são a partir dessas ferramentas combinadas ou não que podem apresentar soluções/saídas para diferentes problemas enfrentados na agricultura, como saúde do solo e das plantas, mudanças climáticas, ervas daninhas e etc.

\section{Internet of things (IoT)}

A internet das coisas, ou em inglês Internet of Things (IoT), é uma técnica que 
combina os recursos existentes na internet para o controle de dispositivos. O conceito inicial de internet das coisas foi introduzido pelo Massachusetts Institute of Technology (MIT) AutoID Labs na década de 1990 e aplicado primeiramente em uma cafeteira no de 1999. Passando alguns meses, também aplicaram pela primeira vez em uma torradeira, em que podia ser ligada e desligada de modo remoto através da internet. Diante disso, ao longo dos anos foram aparecendo diferentes conceitos atribuídos ao IoT, porém, sempre com o mesmo princípio que é a sincronização de objetos por meio da intervenção de pessoas. Figura 01 mostra a relação entre os três aspectos da internet das coisas.

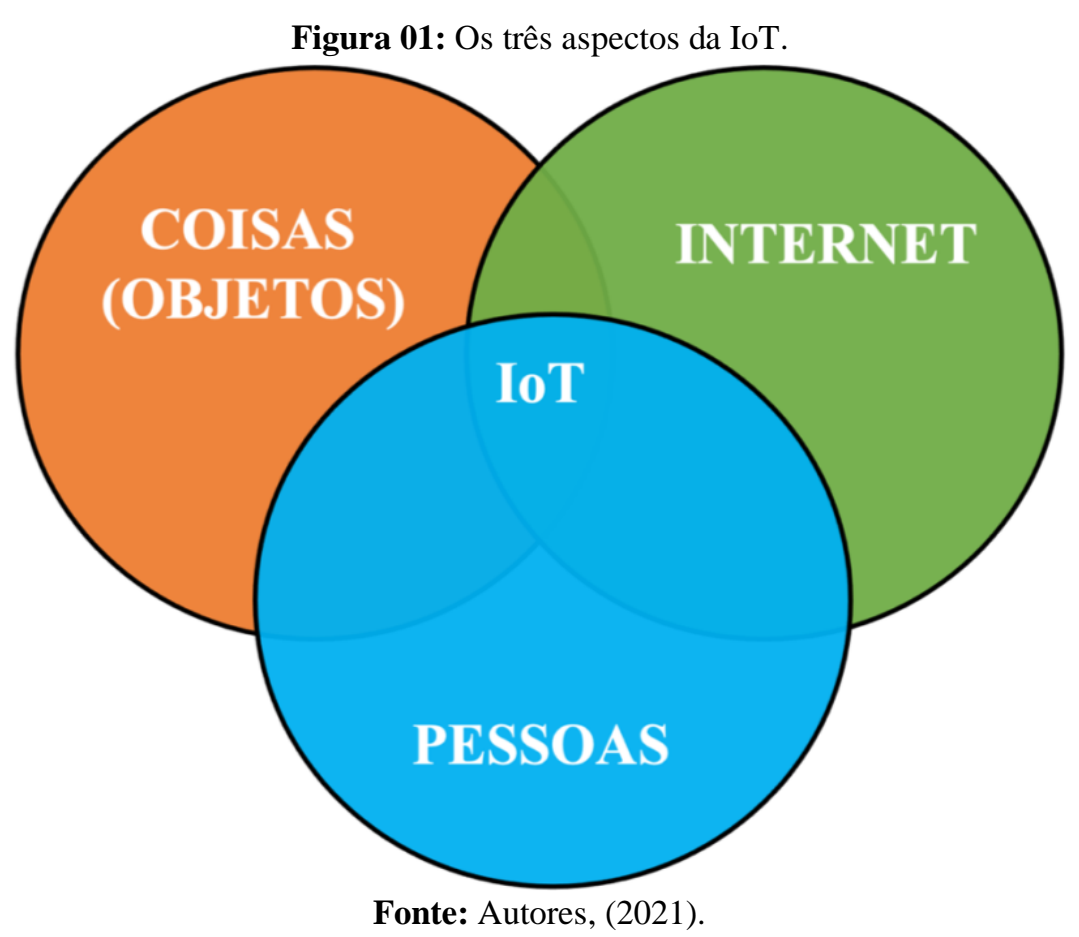

Hoje em dia, graças à internet é possível encaminhar e-mails associados com grande quantidade de conteúdo, anexos e vídeos ou mesmo interagir com pessoas de todo o mundo via redes sociais. Em outros âmbitos, por exemplo o acadêmico, o industrial e o agropecuário, utilizam-se de IoT para o estudo e desenvolvimento de sensores para o controle de variáveis importantes envolvidas em determinado processo/problemática (BANDYOPADHYAY; SEN, 2011). Aplicado na agricultura, encontra-se a IoT inserida em abordagens de gerenciamento agrícola com a finalidade de garantir o desenvolvimento adequado e saudável da planta, sem que use em excesso defensivos agrícolas ou mesmo fertilizantes. Um grupo de pesquisa abordou a integração de redes de controle e de IoT em uma situação real na produção agrícola em estufa, em que propuseram um sistema de monitoramento remoto com internet e comunicações sem fio combinadas (JI-CHUN ZHAO et al., 2010). Por fim, obtiveram ótimos 


\section{AGRICULTURA 4.0: UMA VISÃO GERAL DAS INOVAÇÕES TECNOLÓGICAS}

resultados com a plantação em estufa utilizando a tecnologia, promovendo um excelente crescimento das plantas em estudo.

Uma das inúmeras vantagens do uso da internet das coisas dentro da agricultura é a possibilidade da previsão do desempenho da cultura em diferentes circunstâncias. Assim, o uso de sensores sem fio baseado em IoT podem ser usados para a coleta de diversas variáveis, tais como do clima e da quantidade de fertilizantes/defensivos. O desenvolvimento de um novo design da plataforma SmartFarmNet utilizando IoT foi reportada, em que possibilitou automatizar a coleta de dados ambientais, do solo, fertilização e irrigação (JAYARAMAN et al., 2016). Além de correlacionar automaticamente esses dados e filtrar os dados inválidos da perspectiva da avaliação do desempenho da cultura e calcular as previsões da safra. A plataforma SmartFarmNet possibilita integrar virtualmente qualquer dispositivo IoT, desde sensores, câmeras e até mesmo estações meteorológicas e entre outros disponíveis no mercado para o armazenamento de dados na nuvem e assim analisar. Na Tabela 02 mostra algumas pesquisas científicas baseadas em IoT aplicado na agricultura.

Tabela 02: Lista de alguns trabalhos encontrados na literatura baseados em IoT aplicado na agricultura.

\begin{tabular}{|c|c|c|}
\hline Problemática & Título do trabalho & Referência \\
\hline $\begin{array}{l}\text { Conexão entre } \\
\text { consumidores e } \\
\text { produtores rurais }\end{array}$ & $\begin{array}{c}\text { Internet of things: applications and } \\
\text { challenges in technology and } \\
\text { standardization }\end{array}$ & $\begin{array}{l}\text { (BANDYOPADHY } \\
\text { AY; SEN, 2011) }\end{array}$ \\
\hline $\begin{array}{l}\text { Gerenciamento de } \\
\text { inúmeros } \\
\text { dispositivos na } \\
\text { lavoura }\end{array}$ & $\begin{array}{c}\text { Farm management systems and the future } \\
\text { internet era }\end{array}$ & $\begin{array}{l}\text { (KALOXYLOS et } \\
\text { al., 2012) }\end{array}$ \\
\hline $\begin{array}{l}\text { Otimizar o modo } \\
\text { operacional de coleta } \\
\text { de dados através de } \\
\text { uma plataforma } \\
\text { virtual }\end{array}$ & $\begin{array}{l}\text { Internet of things platform for smart } \\
\text { farming: experiences and lessons learnt }\end{array}$ & $\begin{array}{l}\text { (JAYARAMAN et } \\
\text { al., 2016) }\end{array}$ \\
\hline $\begin{array}{l}\text { Mudanças de } \\
\text { percepções dos } \\
\text { pequenos produtores } \\
\text { rurais com relação } \\
\text { ao uso de } \\
\text { tecnologias }\end{array}$ & $\begin{array}{l}\text { Adoption of precision agriculture } \\
\text { technologies by German crop farmers }\end{array}$ & $\begin{array}{l}\text { (PAUSTIAN; } \\
\text { THEUVSEN, 2017) }\end{array}$ \\
\hline $\begin{array}{l}\text { O uso de modelos } \\
\text { para a análise de } \\
\text { dados }\end{array}$ & $\begin{array}{l}\text { Machine learning applications on } \\
\text { agricultural datasets for smart farm } \\
\text { enhancement }\end{array}$ & $\begin{array}{l}\text { (BALDUCCI; } \\
\text { IMPEDOVO; } \\
\text { PIRLO, 2018) }\end{array}$ \\
\hline
\end{tabular}

Fonte: Autores, (2021). 
Portanto, como observado anteriormente, o conceito de IoT pode ser correlacionado com "coisas" que estão associadas pela internet. Contudo, são muitos os desafios para a sua utilização na lavoura, principalmente em pequenas propriedades rurais que não tem acesso à internet e às tecnologias de ponta. Mas, mesmo assim, a internet das coisas é uma aliada na agricultura 4.0 e na agricultura de precisão, em que se utiliza sensores, bluetooth, imagens e etc para alcançar o aumento sustentável da produção agrícola mundial.

\section{Inteligência artificial e outras tecnologias}

A inteligência artificial (IA) é uma das grandes apostas atualmente no mercado, seja no segmento do varejo ou mesmo no agronegócio. Aliás, o uso de IA no mundo dos negócios está favorecendo muitas empresas, pois através dessa tecnologia permite delinear o perfil e as necessidades dos seus consumidores, em que providenciam produtos e serviços de acordo com o que buscam (POLOZ, 2021). Além de oferecer vantagens financeiras para quem os contrata ou compra algum objeto, mediante uma análise de todas as marcas que estão na rede, ou seja, os seus concorrentes diretos. Diante disso, aplicada na agricultura é possível observar inúmeras finalidades da IA, seja no controle remoto de veículo aéreo não tripulado ou mesmo na predição de chuva e temperatura no campo (LEZOCHE et al., 2020).

A inteligência artificial é caracterizada por um conjunto de ferramentas computacionais e estatísticas para que computadores façam tarefas que exija ações humanas. O termo IA foi incialmente apresentado por John McCarthy na década de 1950 em uma conferência nos EUA (MARCUS, 2004). A IA geralmente é executada mediante um conjunto de ferramentas e áreas, por exemplo, a análise de dados e machine learning. Machine learning (ML) envolve a análise multidisciplinar entre probabilidade, estatística, algoritmo e entre outras teorias (PARTEL; CHARAN KAKARLA; AMPATZIDIS, 2019). Com o progresso e o intenso uso da ferramenta ML, cada vez mais outras áreas estão utilizando modelos para a realização de previsões sobre o futuro e assim, possa facilitar na tomada de decisões racionais com base nessas análises preditivas (BALDUCCI; IMPEDOVO; PIRLO, 2018). Logo, a agricultura que no presente momento necessita aumento contínuo da produção e práticas mais sustentáveis na lavoura, a aplicação de ML e IA são em conjunto uma grande vantagem ao setor. Além disso, a implementação de inteligência artificial na agricultura para a otimização da irrigação e aplicação de pesticidas e herbicidas é reportada constantemente na literatura como uma aliada na reversão de desperdícios de água e defensivos. Na Tabela 03 mostra alguns trabalhos que utilizaram inteligência artificial aplicado na lavoura. 


\section{AGRICULTURA 4.0: UMA VISÃO GERAL DAS INOVAÇÕES TECNOLÓGICAS}

Tabela 03: Lista de alguns trabalhos encontrados na literatura que utilizaram inteligência artificial aplicado na lavoura.

$\begin{array}{lll}\text { Cultura } & \text { Título do trabalho } & \text { Referência }\end{array}$

Cana-de-açúcar

Pimenta e entre outras
Weed detecting robot in sugarcane fields using fuzzy real time classifier

Development and evaluation of a low-cost and smart technology for precision weed management utilizing artificial intelligence

Arroz

Development of small weeding robots for rice fields

Arroz

Arroz
Agricultural intensification and damages to human health in relation to agrochemicals: Application of artificial intelligence
(SUJARITHA et al., 2017)

(PARTEL;

CHARAN

KAKARLA;

AMPATZIDIS, 2019)

(MARUYAMA;

NARUSE, 2014)

(NAKAMURA $e t$ al., 2017)

(ELAHI et al., 2019)

Fonte: Autores, (2021).

Uma temática importante dentro da agricultura é o uso adequado de defensivos, desde que não se torne prejudicial à saúde das plantas e nem das pessoas que os aplicam. Assim, a redução dos impactos dos agroquímicos no meio ambiente e na saúde humana é fundamental para a sustentabilidade agrícola e uma produção mais limpa. Contudo, até o momento, os estudos reportados são limitados e são focados apenas nas questões de intensificação do uso de defensivos nas culturas. Um método de rede neural artificial foi reportado para reduzir a quantidade de pesticidas e nitrogênio puro em 45,2 e 37,2\%, respectivamente, em uma plantação de arroz (ELAHI et al., 2019). Os resultados foram promissores, pois utilizaram de estudos de casos envolvendo agricultores e observaram através de modelos de regressão que o uso de roupas e de óculos de proteção reduziram significativamente a irritação dos olhos e da pele dos aplicadores de pesticidas. Também, a utilização de máscaras diminuiu grandemente os casos de tosse, tontura e náusea. Por fim, com base nos resultados que encontraram sugeriram algumas políticas de boas práticas para a aplicação de agroquímicos no campo, por exemplo, a utilização de sementes melhoradas em que apresentem resistência à diversas pragas. Assim, reduzindo o número de aplicações de defensivos na cultura. 
Também, o uso de robôs está presente em alguns estudos aplicados no segmento agrícola. O desenvolvimento de um robô agrícola de baixo custo para aplicação de fertilizantes e pesticidas na agricultura, além de também exercer o papel de monitoramento da safra foi reportado recentemente (GHAFAR et al., 2021). O protótipo apresentado é um robô de duas rodas que consistiu em uma base móvel, um mecanismo de pulverização, um controlador sem fio para o movimento do robô e uma câmera para monitorar a saúde e o crescimento da cultura, além de detectar a presença de pragas no campo agrícola. Os pesquisadores observaram que nos testes de produtividade realizados com o protótipo foi ligeiramente inferior à de um funcionário humano, porém, a economia de mão de obra proporcionada pelo robô é muito maior, pois o mesmo funciona completamente no modo autônomo. Além disso, o sistema proporcionou maior economia de recursos e redução da contaminação de águas subterrâneas, atingindo os objetivos inerente à agricultura de precisão. Também, o protótipo apresentou excelente duração da bateria. Com isso, percebe-se que o uso de robótica é um ponto positivo e apresenta inúmeras soluções inerente à agricultura, como a reversão da falta de mão de obra, redução dos custos com defensivos e o uso adequado das quantidades de agroquímicos.

Por fim, percebe-se que o uso de IA atrelado às ferramentas tecnológicas é vantajoso na agricultura, principalmente no monitoramento através de drones, sensores e modelos preditivos. Também, com a inserção de robótica no campo pode facilitar a busca por soluções sustentáveis e o aumento de produtividade em pouco espaço, reduzindo o desperdício e os custos operacionais. No entanto, a utilização dessas tecnologias ainda está restrita aos grandes produtores e empresas do ramo. Ou mesmo, ainda não são adequadas para operar na realidade, sendo necessário mais testes e estudos, por exemplo, os robôs.

\section{POTENCIAIS E LIMITAÇÕES}

O produtor rural brasileiro e de países emergentes, os quais são considerados atualmente a grande aposta para o aumento da produtividade de alimentos para suprir as necessidades da população mundial, ainda não presenciam na sua maioria a conectividade (internet) em suas propriedades. Isso, é um grande desafio para a inserção das tecnologias inerentes à agricultura 4.0, pois na maior parte é necessário a integração de computadores e sensores através desse recurso. No entanto, grandes empresas vêm unindo forças para suprir essa necessidade e que isso não seja um fator limitante ao pequeno agricultor utilizar as tecnologias 4.0. Também, há iniciativas que promovem o desenvolvimento de empresas de tecnologia voltadas para o agronegócio (Agritechs), principalmente para que desenvolvam 


\section{AGRICULTURA 4.0: UMA VISÃO GERAL DAS INOVAÇÕES TECNOLÓGICAS}

ferramentas computacionais para o monitoramento de lavouras e facilitem na tomada de decisão do pequeno agricultor. As vantagens do uso de tecnologias da agricultura 4.0 são diversas, como (LEZOCHE et al., 2020):

- Aumento da produtividade;

- Monitoramento das atividades agrícolas por meio de sensores, georreferenciamento, veículo aéreo não tripulado e etc;

- Redução dos custos e dos desperdícios de defensivos e fertilizantes na lavoura;

- Facilidade na tomada de decisão;

- Otimização da gestão do negócio;

- Longevidade do uso do solo;

- Desenvolvimento de modelos preditivos que envolvam a performance da cultura frente às diversas variáveis, como o clima e solo; e

- Acompanhamento da plantação à distância.

Apesar de inúmeros pontos positivos do uso de tecnologia 4.0 na agricultura, também existem limitações, como:

- Custos de implantação;

- Falta de internet no local;

- Adequação das ferramentas à propriedade;

- Falta de profissionais qualificados dentro da área de tecnologia 4.0 e especificamente na agricultura; e

- Dificuldade na análise de dados.

Assim, percebe-se que são diversos os desafios presentes para a implementação de recursos tecnológicos no campo, porém, essas limitações podem ser revertidas em um curto prazo em retorno financeiro ao produtor e entre outros pontos positivos ao meio ambiente/sociedade em geral, tais como o uso consciente da água na irrigação, tratamento adequado ao solo e redução do uso de defensivos agrícolas. Além disso, os pontos positivos do manejo sustentável na agricultura integrado com as tecnologias atuais podem atingir diretamente e/ou indiretamente na diminuição das embalagens agrícolas e em iniciativas sociais para as comunidades rurais.

\section{CONCLUSÃO}

Os benefícios da agricultura 4.0 são perceptíveis ao longo da revisão e é possível visualizar através desse progresso um mundo em que as lavouras apresentem maior 
produtividade e consequentemente, seja possível suprir as necessidades da população mundial. Contudo, ainda é necessário tornar essas ferramentas sustentáveis e viáveis para os produtores, principalmente às pequenas propriedades. Diante disso, o Brasil como um dos principais produtores de alimentos do mundo tem papel importante nesse progresso agrícola, pois sua adesão a essas tecnologias possibilitará aumentar a atual performance da produtividade agrícola e a modernização das lavouras em território nacional. Além disso, hoje em dia percebe-se uma adesão gradual dos proprietários rurais quanto ao uso dessas tecnologias e assim, à medida que aumentar o número de usuários dessas ferramentas são desmistificados os pré-conceitos que estão presentes nessa temática.

\section{REFERÊNCIAS}

AFOLALU, S. A.; IKUMAPAYI, O. M.; ABDULKAREEM, A.; SOETAN, S. B.; EMETERE, M. E.; ONGBALI, S. O. Enviable roles of manufacturing processes in sustainable fourth industrial revolution - A case study of mechatronics. Materials Today: Proceedings, 2021. Disponível em: https://doi.org/10.1016/j.matpr.2021.01.099

ANGOM, J.; VISWANATHAN, P. K.; RAMESH, M. V. The dynamics of climate change adaptation in India: A review of climate smart agricultural practices among smallholder farmers in Aravalli district, Gujarat, India. Current Research in Environmental Sustainability, v. 3, p. 100039, 2021. Disponível em: https://doi.org/10.1016/j.crsust.2021.100039

BALDUCCI, F.; IMPEDOVO, D.; PIRLO, G. Machine Learning Applications on Agricultural Datasets for Smart Farm Enhancement. Machines, v. 6, n. 3, p. 38, 2018. Disponível em: https://doi.org/10.3390/machines6030038

BANDYOPADHYAY, D.; SEN, J. Internet of things: Applications and challenges in technology and standardization. Wireless Personal Communications, v. 58, n. 1, p. 49-69, 2011. Disponível em: https://doi.org/10.1007/s11277-011-0288-5

BJORNLUND, V.; BJORNLUND, H. Understanding agricultural water management in a historical context using a socioeconomic and biophysical framework. Agricultural Water Management, v. 213, n. November 2017, p. 454-467, 2019. Disponível em: https://doi.org/10.1016/j.agwat.2018.10.037

ELAHI, E.; WEIJUN, C.; ZHANG, H.; NAZEER, M. Agricultural intensification and damages to human health in relation to agrochemicals: Application of artificial intelligence. Land Use Policy, v. 83, n. February, p. 461-474, 2019. Disponível em: https://doi.org/10.1016/j.landusepol.2019.02.023

FUENTES-LLANILLO, R.; TELLES, T. S.; SOARES JUNIOR, D.; DE MELO, T. R.; FRIEDRICH, T.; KASSAM, A. Expansion of no-tillage practice in conservation agriculture in Brazil. Soil and Tillage Research, v. 208, n. June 2020, p. 104877, 2021. Disponível em: 
https://doi.org/10.1016/j.still.2020.104877

GHAFAR, A. S. A.; HAJJAJ, S. S. H.; GSANGAYA, K. R.; SULTAN, M. T. H.; MAIL, M. F.; HUA, L. S. Design and development of a robot for spraying fertilizers and pesticides for agriculture. Materials Today: Proceedings, 2021. Disponível em: https://doi.org/10.1016/j.matpr.2021.03.174

JAYARAMAN, P.; YAVARI, A.; GEORGAKOPOULOS, D.; MORSHED, A.; ZASLAVSKY, A. Internet of Things Platform for Smart Farming: Experiences and Lessons Learnt. Sensors, v. 16, n. 11, p. 1884, 2016. Disponível em: https://doi.org/10.3390/s16111884

JI-CHUN ZHAO; JUN-FENG ZHANG; YU FENG; JIAN-XIN GUO. The study and application of the IOT technology in agriculture. In: 2010, 2010 3rd International Conference on Computer Science and Information Technology. : IEEE, 2010. p. 462-465. Disponível em: https://doi.org/10.1109/ICCSIT.2010.5565120

JONES, J. W. et al. Brief history of agricultural systems modeling. Agricultural Systems, v. 155, p. 240-254, 2017. Disponível em: https://doi.org/10.1016/j.agsy.2016.05.014

JUNG, J.; MAEDA, M.; CHANG, A.; BHANDARI, M.; ASHAPURE, A.; LANDIVARBOWLES, J. The potential of remote sensing and artificial intelligence as tools to improve the resilience of agriculture production systems. Current Opinion in Biotechnology, v. 70, p. 15-22, 2021. Disponível em: https://doi.org/10.1016/j.copbio.2020.09.003

KAKANI, V.; NGUYEN, V. H.; KUMAR, B. P.; KIM, H.; PASUPULETI, V. R. A critical review on computer vision and artificial intelligence in food industry. Journal of Agriculture and Food Research, v. 2, n. February, p. 100033, 2020. Disponível em: https://doi.org/10.1016/j.jafr.2020.100033

KALOXYLOS, A. et al. Farm management systems and the Future Internet era. Computers and Electronics in Agriculture, v. 89, p. 130-144, 2012. Disponível em: https://doi.org/10.1016/j.compag.2012.09.002

KAMILARIS, A.; KARTAKOULLIS, A.; PRENAFETA-BOLDÚ, F. X. A review on the practice of big data analysis in agriculture. Computers and Electronics in Agriculture, v. 143, n. September, p. 23-37, 2017. Disponível em: https://doi.org/10.1016/j.compag.2017.09.037

LEZOCHE, M.; PANETTO, H.; KACPRZYK, J.; HERNANDEZ, J. E.; ALEMANY DÍAZ, M. M. E. Agri-food 4.0: A survey of the Supply Chains and Technologies for the Future Agriculture. Computers in Industry, v. 117, p. 103187, 2020. Disponível em: https://doi.org/10.1016/j.compind.2020.103187

MARCUS, M. The 2003 Benjamin Franklin Medal in computer and cognitive science presented to John McCarthy (Stanford California). John McCarthy's multiple contributions to the foundations of artificial intelligence and computer science. Journal of the Franklin Institute, v. 341, n. 3, p. 215-221, 2004. Disponível em: https://doi.org/10.1016/j.jfranklin.2003.12.023 
MARUYAMA, A.; NARUSE, K. Development of small weeding robots for rice fields. In: 2014, 2014 IEEE/SICE International Symposium on System Integration. : IEEE, 2014. p. 99-105. Disponível em: https://doi.org/10.1109/SII.2014.7028019

MEYER, G. .; CAMARGO NETO, J.; JONES, D. D.; HINDMAN, T. W. Intensified fuzzy clusters for classifying plant, soil, and residue regions of interest from color images. Computers and Electronics in Agriculture, v. 42, n. 3, p. 161-180, 2004. Disponível em: https://doi.org/10.1016/j.compag.2003.08.002

NAKAMURA, K.; KIMURA, M.; ANAZAWA, T.; TAKAHASHI, T.; NARUSE, K. Investigation of weeding ability and plant damage for rice field weeding robots. SII 2016 2016 IEEE/SICE International Symposium on System Integration, p. 899-905, 2017. Disponível em: https://doi.org/10.1109/SII.2016.7844114

PARTEL, V.; CHARAN KAKARLA, S.; AMPATZIDIS, Y. Development and evaluation of a low-cost and smart technology for precision weed management utilizing artificial intelligence. Computers and Electronics in Agriculture, v. 157, n. January, p. 339-350, 2019. Disponível em: https://doi.org/10.1016/j.compag.2018.12.048

PAUSTIAN, M.; THEUVSEN, L. Adoption of precision agriculture technologies by German crop farmers. Precision Agriculture, v. 18, n. 5, p. 701-716, 2017. Disponível em: https://doi.org/10.1007/s11119-016-9482-5

POLOZ, S. S. Technological progress and monetary policy: Managing the fourth industrial revolution. Journal of International Money and Finance, p. 102373, 2021. Disponível em: https://doi.org/10.1016/j.jimonfin.2021.102373

SAHU, K. K.; MISHRA, A. K.; LAL, A.; SAHU, S. A. India Fights Back: COVID-19 Pandemic. Heart \& Lung, v. 49, n. 5, p. 446-448, 2020. Disponível em: https://doi.org/10.1016/j.hrtlng.2020.04.014

SCHNASE, J. L.; DUFFY, D. Q.; TAMKIN, G. S.; NADEAU, D.; THOMPSON, J. H.; GRIEG, C. M.; MCINERNEY, M. A.; WEBSTER, W. P. MERRA Analytic Services: Meeting the Big Data challenges of climate science through cloud-enabled Climate Analyticsas-a-Service. Computers, Environment and Urban Systems, v. 61, p. 198-211, 2017. Disponível em: https://doi.org/10.1016/j.compenvurbsys.2013.12.003

SUJARITHA, M.; ANNADURAI, S.; SATHEESHKUMAR, J.; KOWSHIK SHARAN, S.; MAHESH, L. Weed detecting robot in sugarcane fields using fuzzy real time classifier. Computers and Electronics in Agriculture, v. 134, p. 160-171, 2017. Disponível em: https://doi.org/10.1016/j.compag.2017.01.008

TALAVIYA, T.; SHAH, D.; PATEL, N.; YAGNIK, H.; SHAH, M. Implementation of artificial intelligence in agriculture for optimisation of irrigation and application of pesticides and herbicides. Artificial Intelligence in Agriculture, v. 4, p. 58-73, 2020. Disponível em: https://doi.org/10.1016/j.aiia.2020.04.002

TRIPATHI, S.; SRINIVAS, V. V.; NANJUNDIAH, R. S. Downscaling of precipitation for climate change scenarios: A support vector machine approach. Journal of Hydrology, v. 330, n. 3-4, p. 621-640, 2006. Disponível em: https://doi.org/10.1016/j.jhydrol.2006.04.030 
VELMURUGAN, P.; KANNAGI, A.; VARSHA, M. Superior fuzzy enumeration crop prediction algorithm for big data agriculture applications. Materials Today: Proceedings, 2021. Disponível em: https://doi.org/10.1016/j.matpr.2021.02.578

ZHANG, L.; GUAN, Y.; JIANG, S. C. Investigations of soil autotrophic ammonia oxidizers in farmlands through genetics and big data analysis. Science of The Total Environment, v. 777, p. 146091, 2021. Disponível em: https://doi.org/10.1016/j.scitotenv.2021.146091

ZILLI, M.; SCARABELLO, M.; SOTERRONI, A. C.; VALIN, H.; MOSNIER, A.; LECLÈRE, D.; HAVLÍK, P.; KRAXNER, F.; LOPES, M. A.; RAMOS, F. M. The impact of climate change on Brazil's agriculture. Science of The Total Environment, v. 740, p. 139384, 2020. Disponível em: https://doi.org/10.1016/j.scitotenv.2020.139384 